\title{
MBGEM: a stack of borated GEM detector for high efficiency thermal neutron detection
}

\author{
A. Muraro ${ }^{5,6,10}$, G. Claps ${ }^{1,4}$, G. Croci ${ }^{5,6,10}$, C. C. Lai $^{3,8}$, R. De Oliveira ${ }^{2}$, S. Altieri ${ }^{7}$,

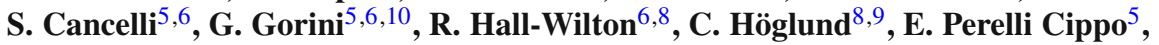 \\ L. Robinson ${ }^{8}$, P. Svensson ${ }^{8}$, F. Murtas ${ }^{1,2, a}{ }^{1}$ \\ ${ }^{1}$ Laboratori Nazionali di Frascati-INFN, Frascati, Italy \\ 2 CERN, 1211 Geneva, Switzerland \\ 3 Linköping University, Linköping, Sweden \\ ${ }^{4}$ Centro Ricerche Frascati (ENEA), Frascati, Italy \\ 5 Istituto per la Scienza e Tecnologia dei Plasmi (ISTP) (CNR), Milan, Italy \\ 6 Dipartimento di Fisica “G. Occhialini”, Università degli Studi di Milano - Bicocca, Milan, Italy \\ 7 Dip. di Fisica and INFN, Università degli Studi di Pavia, Pavia, Italy \\ ${ }^{8}$ Detector Group, European Spallation Source ERIC (ESS), Lund, Sweden \\ ${ }^{9}$ Impact Coatings AB, Linköping, Sweden \\ 10 INFN, Sezione di Milano - Bicocca, Milan, Italy
}

Received: 30 March 2021 / Accepted: 24 June 2021

(C) The Author(s) 2021

\begin{abstract}
A new position-sensitive thermal neutron detector based on boron-coated converters has been developed as an alternative to today's standard ${ }^{3} \mathrm{He}$-based technology for application to thermal neutron scattering. The key elements of the development are the boron-coated GEM foils (Sauli in Nucl Instrum Methods Phys Res Sect A Accel Spectrom Detect Assoc Equip 386:531, 1997) that are used as a multi-layer neutron converter via the ${ }^{10} \mathrm{~B}(n, \alpha){ }^{7} \mathrm{Li}$ reaction together with an efficient collection of the produced secondary electrons. This paper reports the test performed on a 3 layers converter prototype coupled to a GEMPix detector (Murtas in Radiat Meas 138:106421, 2020), carried out in order to study the possibility to produce a large-scale multi-layer neutron detector capable to reach high detection efficiency with high spatial resolution and able to sustain the high neutron flux expected in the new neutron spallation source under development like the ESS.
\end{abstract}

\section{Introduction}

The recent ${ }^{3} \mathrm{He}$ shortage [3], which is due to a progressive nuclear disarmament started in 2000 , has resulted in reduced availability of this noble gas and a consequent high increase in its price. Nowadays the world's most important suppliers (USA and Russia) are keeping the residual amount of ${ }^{3} \mathrm{He}$ mainly for homeland security and nuclear safeguards applications. As a consequence, the availability of ${ }^{3} \mathrm{He}$ gas for thermal neutron detectors (in the form of high-pressure single-wire proportional counter tubes) has been severely reduced. On the other hand, the scientific community is experiencing the need to realize new large area positionsensitive devices able to fully exploit the increase of neutron flux offered by new spallation

\footnotetext{
a e-mail: fabrizio.murtas@ cern.ch (corresponding author)
} 
neutron sources like ESS. These two reasons led to the development of new position-sensitive neutron detectors capable of detection efficiencies comparable with ${ }^{3} \mathrm{He}$ tubes, but being able to sustain much higher counting rates than the $30-50 \mathrm{kHz}$ typical for single ${ }^{3} \mathrm{He}$ tubes [4]. A possible approach relies on the use of solid neutron converters (such as boron or lithium) combined to charged particle detectors, like the gas electron multiplier (GEM) [1] detector. GEM detectors are typically used for tracking and triggering application in high energy physics [5], but they can also be used for neutral particles detection like fast [6-9] and thermal $[10-14]$ neutrons or photon $[15,16]$. Neutral particles detection with GEM is typically performed by adopting a customized cathode configuration, where primary electrons are liberated in the detector gas due to neutrals interactions, followed by an amplification stage (composed by one or multiple GEM foils). Finally, the motion of the multiplied electrons in the induction region induces a signal on an anode (that can be fully customized for the specific application) read out by a dedicated data acquisition system (DAQ). This paper describes the development, the construction and the characterization of a new GEM-based thermal neutron detector called MBGEM (multi-boron GEM), equipped with a converter cathode made of a series of GEM foils coated on both sides with a layer ( $1 \mu \mathrm{m}$ thick) of ${ }^{10} \mathrm{~B}_{4} \mathrm{C}$. The paper is addressed to study the response of the new converter configuration in view of the production of a large area, high efficiency thermal neutron detector.

\section{The MBGEM principle}

The efficiency $(\epsilon)$ of a thermal neutron detector based on a solid-state converter (such as ${ }^{10} \mathrm{~B}$ or ${ }^{6} \mathrm{Li}$ ) combined to a charged particle amplifier can be defined as the result of the product of three factors:

- the neutron interaction probability $(p)$,

- the escape probability $(\xi)$ of the charged products generated by the neutron nuclear reaction

- the extraction efficiency $(\eta)$ of the primary charge liberated by the reaction products.

In case of boron-based converters the nuclear reaction ${ }^{10} \mathrm{~B}(n, \alpha){ }^{7} \mathrm{Li}$ is exploited whose cross section is about $3844 b$ for $25 \mathrm{meV}$ neutrons. This reaction is characterized by two main channels:

$$
\begin{aligned}
& { }^{10} \mathrm{~B}+n \rightarrow L i+\alpha(Q \text { Value }=2.792 \mathrm{MeV}, \text { ground state }) ; \text { Branching Ratio }=6 \% \\
& { }^{10} \mathrm{~B}+n \rightarrow 7 L i^{\star}+\alpha \\
& +\gamma(0.478 \mathrm{MeV})(Q \text { Value }=2.310 \mathrm{MeV}, \text { excited state }) ; \text { Branching Ratio }=94 \% .
\end{aligned}
$$

Therefore when thermal neutrons interact with boron, the reaction leads to ${ }^{7} \mathrm{Li}$ in the excited state $94 \%$ of the times, while the remaining $6 \%$ are ground state ${ }^{7} \mathrm{Li}$. Conservation of energy and momentum gives $E_{\mathrm{Li}}=0.84 \mathrm{MeV}$ and $E_{\alpha}=1.47 \mathrm{MeV}$ for the excited state reaction, and $E_{\mathrm{Li}}=1.16 \mathrm{MeV}, E_{\alpha}=1.78 \mathrm{MeV}$ for the ground state reaction, and implies that the two charged particles $\left({ }^{7} \mathrm{Li}\right.$ and $\left.\alpha\right)$ are emitted back to back. It means that at least one of the (charged) reaction products is likely to be revealed into the adjacent detector. In addition when ${ }^{7} \mathrm{Li}$ is left in the excited state, there is always an emission of $478 \mathrm{keV} \gamma$ ray.

The challenge in the design of novel detectors relies on finding a geometrical and electrical configuration able to simultaneously optimize $p, \xi$ and $\eta$ parameters. The probability $p$ is related to the macroscopic neutron (absorption) cross section of the converter material used $\left({ }^{10} \mathrm{~B}_{4} \mathrm{C}\right.$ in the case of this paper), while $\xi$ is related to the range of the produced charge particles 
in the converter layer itself. Probability $p$ can be increased by using a thicker converter layer, but this decreases the probability that the nuclear charged products can reach the gas. The $\alpha$ and ${ }^{7} \mathrm{Li}$ ions are emitted isotropically along the ${ }^{10} \mathrm{~B}_{4} \mathrm{C}$ layer thickness and one or other of these particles may leave the ${ }^{10} \mathrm{~B}_{4} \mathrm{C}$ layer and ionize the gas volume in which the converter is positioned. Therefore the charge escape probability $\xi$ depends on:

- the neutron interaction point along the thickness of the ${ }^{10} \mathrm{~B}_{4} \mathrm{C}$ layer;

- the direction of emission of the charged particles;

- the ${ }^{10} \mathrm{~B}_{4} \mathrm{C}$ layer thickness.

The charged particles escaping from the layer will feature a continuous energy spectrum ranging from 0 to $E_{\alpha}$ and $E_{\mathrm{Li}}$. The typical range of alpha and lithium ions in ${ }^{10} \mathrm{~B}_{4} \mathrm{C}$ is about $3.4 \mu \mathrm{m}$ and $1.7 \mu \mathrm{m}$, respectively [17]. Therefore a single converter thicker than $2 \mu \mathrm{m}$ will imply a large loss of detection efficiency (mainly for the $L_{i}$ ions) due to $\xi$ reduction. A possible approach to maximize the detection efficiency is the use of multiple stacked thin converter layers that must be able to be transparent to the secondary electrons produced by the gas ionization due to alpha and lithium ions, thus assuring a high extraction probability $\eta$. A converter thickness of $1 \mu \mathrm{m}$ of ${ }^{10} \mathrm{~B}_{4} \mathrm{C}$ it seems the final good solution as reported also in other works based on a calculated optimization of neutron detection efficiency [18].

\subsection{The preparation of the GEM foil}

The key element of the present study is the double-face ${ }^{10} \mathrm{~B}_{4} \mathrm{C}$-coated GEM foil (called BGEM borated GEM) used to detect neutrons via the ${ }^{10} \mathrm{~B}(n, \alpha){ }^{7} \mathrm{Li}$ reaction. A GEM [19] is made of a thin $(50 \mu \mathrm{m})$ Kapton foil, copper clad ( $5 \mu \mathrm{m}$ thick) on each side, perforated with high surface density of holes, each one acting as an electron multiplication channel. Each hole has a bi-conical structure with an external (internal) diameter of $70 \mu \mathrm{m}(50 \mu \mathrm{m})$ and a pitch of $140 \mu \mathrm{m}$. The bi-conical shape of the hole minimizes the effect of charging-up of the Kapton inside the holes and is a consequence of the double-mask process used in standard photolithographic technologies. A typical voltage difference of $250-500 \mathrm{~V}$ is applied between the two copper sides, giving fields as high as $150 \mathrm{kV} / \mathrm{cm}$ into the holes, resulting in an electron multiplication up to a few thousands.

The BGEM foil is produced by introducing a modification to the standard GEM production method. The base material is the usual $50 \mu \mathrm{m}$-thick polyimide foil cladded on both sides with copper $(5 \mu \mathrm{m})$. The standard pattern of holes with a diameter of $70 \mu \mathrm{m}$ and a pitch of $140 \mu \mathrm{m}$ have been designed on a square of $30 \times 30 \mathrm{~mm}^{2}$ both on top and bottom sides of the foil, followed by a photoresist lamination on both faces. Then photoresist dots have been put covering precisely the copper openings followed by the curing. Afterward these foils were individually sandwiched in Milano Bicocca University with two Al masks that have a $30 \times 30 \mathrm{~mm}^{2}$ opening on each face aligned with the patterned active areas on both side of the foil as shown in Fig. 1.

\subsection{The boron carbide deposition}

The deposition process for coating-enriched amorphous boron carbide ${ }^{10} \mathrm{~B}_{4} \mathrm{C}$ layers on the polyimide foils was carried out since 2015 by ESS Linköping Detector Coatings Workshop, located at Linköping, Sweden. In the following ${ }^{10} \mathrm{~B}$ enrichment means $>95 \%$. The facility is installed with an industrial deposition unit (CC800/9, CemeCon AG) equipped with four direct-current magnetron sputtering cathodes and a sample loading system for batch production. Details about the deposition unit and process development for neutron detector coatings of other types have been published elsewhere [17,20]. 

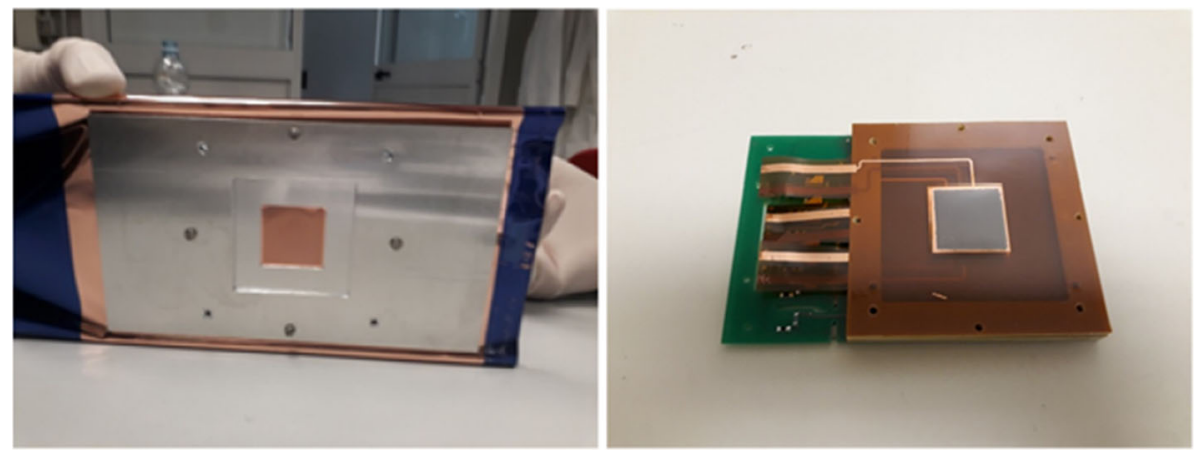

Fig. 1 On the left the GEMPix foil prepared with the aluminum mask before the boron deposition. On the right the final assembling of the multi-layer neutron converter with the electrode detail

The masks realized in Milan ensure good thermal and electrical contact over the surface of the foils to the grounded sample table of the deposition machine, so the foils have less chance to be thermally or electrically damaged during the deposition process. No other treatment, e.g., cleaning, was done to the masked samples prior to the loading of the samples to avoid damages to the photoresist dots.

The deposition chamber was first pumped down to a background pressure of $\sim 1.5 \times$ $10^{-4} \mathrm{~Pa}$, while the samples were heated up to $\sim 100^{\circ} \mathrm{C}$. Then the surfaces of the samples were treated with radiofrequency plasma etching in pure Ar atmosphere at $0.35 \mathrm{~Pa}$ to remove native oxides built up on the $\mathrm{Cu}$ surface and to create higher surface roughness for better film adhesion. After the etching, the samples were subsequently coated with a ${ }^{10} \mathrm{~B}_{4} \mathrm{C}$ layer in pure $\mathrm{Ar}$ at $\sim 0.40 \mathrm{~Pa}$ and with a Cu capping layer in pure $\mathrm{Ar}$ at $\sim 0.30 \mathrm{~Pa}$ without breaking the vacuum.

The selection of the working pressure for the ${ }^{10} \mathrm{~B}_{4} \mathrm{C}$ layer is mainly a compromise between the residual compressive stress and the increased oxygen content due to a relatively lowtemperature process, which has been systematically studied on Si substrates [21]. A simple scratch test has been carried out with tweezers on non-active areas of coated foils, while only the surface of the $\mathrm{Cu}$ capping layers can be scratched off due to its softness. No spontaneous spall-off of the ${ }^{10} \mathrm{~B}_{4} \mathrm{C}$ films was observed either throughout the GEM foils production. The thickness of the coatings was measured with a profilometer (DektakXT, Bruker) on a $\mathrm{Si}(100)$ thickness reference mounted at an equivalent position of the deposition chamber in the same deposition run. The overall thickness of the ${ }^{10} \mathrm{~B}_{4} \mathrm{C}$ plus $\mathrm{Cu}$ layer was measured to be $\sim 4.0 \mu \mathrm{m}$ on a $\mathrm{Si}(100)$ thickness. The individual thickness of the ${ }^{10} \mathrm{~B}_{4} \mathrm{C}$ layer and the $\mathrm{Cu}$ layer was calculated to be $\sim 1.0 \mu \mathrm{m}$ and $\sim 3.0 \mu \mathrm{m}$, respectively, according to the deposition rates determined from single material depositions done with the same deposition parameters beforehand. The chemical composition of the ${ }^{10} \mathrm{~B}_{4} \mathrm{C}$ layer was done by elastic recoil detection analysis in the Tandem Laboratory in Uppsala University, while details on the employed detector set-up can be found in [22] and a discussion of the method and statistical and systematic uncertainties in [23,24]. The measurement was carried out on the same Si reference of the deposition rate determination to avoid the thick $\mathrm{Cu}$ capping layer on the samples. The result shows a relative atomic ratio of about $77 \% \mathrm{~B}, 18 \% \mathrm{C}$, and $5 \%$ impurities (mainly $\mathrm{O}$ and $\mathrm{H}$ ). 


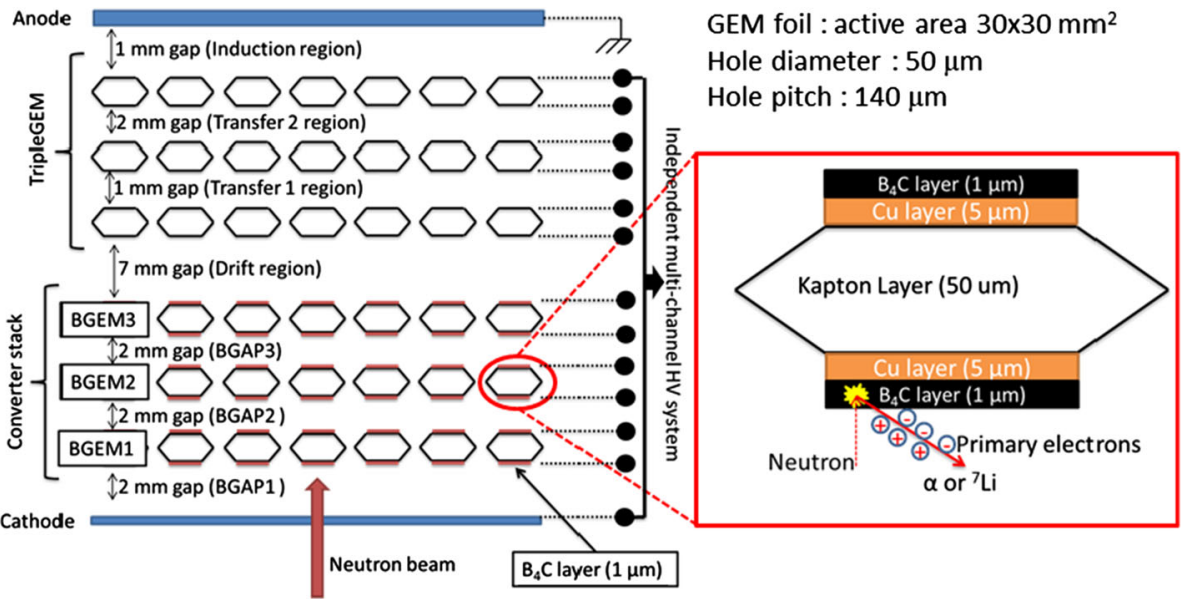

Fig. 2 Schematics of the multi-boron detector. Three BGEM borated GEM foils are stacked and used as neutron converter. Neutron beam impinges orthogonally on the converter and interacts with ${ }^{10} \mathrm{~B}$ via the ${ }^{10} \mathrm{~B}(n, \alpha){ }^{7} \mathrm{Li}$ reaction. One of the two charged products is able to leave the ${ }^{10} \mathrm{~B}_{4} \mathrm{C}$ layer and ionize the gas in which the converter is placed (sketched in the zoomed detail on the right). Electrons liberated by this ionization are able to drift along the converter and reach the triple GEM structure where they are multiplied in order to induce a signal on the pixelated anode constituted by a quad-Timepix chip

\subsection{The final BGEM production}

After the boron deposition, the GEMPix foils came back to CERN for final working and etching. The photoresist dots were stripped by the foils with the aim to etch the polyimide, masked at this point by the boron deposition. After this procedure for the creation of the holes, the foils appear to have on both faces the usual GEM pattern in the central area and copper cladded on the remaining surface. The external copper is then etched to create the electrodes for the voltage application as shown in Fig. 1. The cleaning and the final electrical test ends the operations on the coated foils, to be ready for the detector assembly. All the six BGEMs produced passed the electrical test, and three of them were used for the detector construction.

\subsection{The detector construction}

Three GEMPix foils with different electrodes configuration useful for the final HV distribution (see Fig. 1) have been assembled. The gluing of these foils on FR4 frames and the final assembling of the multi-layer neutron converter were performed in a clean room of Laboratori Nazionali di Frascati of INFN.

The multi-boron GEMPix detector is schematically represented in Fig. 2.

The detector has a neutron converter region, composed by a PCB with gold-plated $30 \times$ $30 \mathrm{~mm}^{2}$ cathode followed by a stack of 3 BGEM foils. Each electrode of the 3 GEM foils is connected to an independent channel of the HV system [25], so that the electric fields and gain can be set individually on each GEM foil. Neutrons enter in the detector orthogonally to the cathode. They can be absorbed in one of the six $1 \mu \mathrm{m}$ thick ${ }^{10} \mathrm{~B}_{4} \mathrm{C}$ layers of the converter stack (two ${ }^{10} \mathrm{~B}_{4} \mathrm{C}$ layers for each BGEM foil) via the ${ }^{10} \mathrm{~B}(n, \alpha){ }^{7} \mathrm{Li}$ reaction. The two created charged particles are emitted back to back (momentum conservation), so only one of them can escape from the ${ }^{10} \mathrm{~B}_{4} \mathrm{C}$ layer and ionize the detector gas (a mixture of $\mathrm{Ar}_{\mathrm{CO}_{2}} \mathrm{CF}_{4} 45 / 15 / 40$ ), 
producing the secondary electrons that will be driven by the applied electric field toward to the holes of the GEM foils, where they are transported and eventually multiplied subjected to the high electric field dipole present in the hole. The HV configuration of the detector must ensure a uniform response independently from the neutron interaction point in the converter stack. The gain of the BGEM composing the converter stack must be close to one, so that the charge will be transfer from one conversion region to another without multiplication.

The ionization charge produced by the multi-boron converter by the single particle is then drifted toward the triple GEM amplifier where will be finally multiplied by a factor $10^{2}-10^{4}$ depending of the applied voltage generated by the HVGEM module [26]. Below the triple GEM structure at $1 \mathrm{~mm}$ distance a pixelated readout is organized using a matrix of $2 \times 2$ 'naked' Timepix ASICs [27] with an active area of the detector equal to $3 \times 3 \mathrm{~cm}^{2}$. The movement of the charge in the induction region induces a signal on a group of pads of the Timepix ASIC (dimension of each pad is $55 \times 55 \mu \mathrm{m}^{2}$ ), underlying the so-called charge cluster.

The Timepix ASICs are operated in Time-over-Threshold (ToT) mode and read out by the FITPix [28] (an FPGA-based module) using the Pixelman software [29]. In ToT mode, the Timepix measures the time spent above a fixed threshold by the analogue signal induced on the single pixel, which is proportional to the collected charge and then (after calibration) to the energy deposited in the detector by the radiation. The measurement of the deposited charge (call event) is done for an adjustable time window, here called "exposure time." For the scope of this study, the exposure time must be set with the goal to obtain a limited number of well-separated charge clusters in each event, so that the response to each single interacting particle can be studied. An online cluster analysis algorithm is used in order to produce a file output with the list of the reconstructed charge clusters, reporting for each one the main information such as the start time of the event, the total number of detected clusters, the total charge and some other geometric parameters.

\section{Detector characterization}

The detector was characterized under X-ray and gamma irradiation at the CERN and under neutron irradiation at the LENA reactor in Pavia [30].

\subsection{Optimization of the converter charge extraction efficiency}

As highlighted in par. 2, the fundamental parameter of the MBGEM detector is the capability to extract the primary charge created by the incoming radiation from the converter stack. Ideally, the response of the detector should be the same independently from the neutron interaction point in the converter.

Thanks to the independent HV control applied to each BGEM, it is possible to experimentally tune the gain of the BGEMs in order to obtain a uniform response. This optimization was done by irradiating with a ${ }^{55} \mathrm{Fe}$ radioactive source each single gas gap (Bgap) between BGEM foils through small apertures on the frames side. In this way X-rays impinge laterally on the detector and get collimated by the small frame aperture (see Fig. 3).

Figure 4 shows a typical charge spectrum recorded when X-rays convert only in the drift region. As expected, both the main peak $(5.9 \mathrm{keV})$ and the escape peak $(3 \mathrm{keV})$ are clearly visible. Since the X-rays enter the detector in the drift region (i.e., between the triple GEM and the converter) electrons produced in this region by X-rays ionization move toward the triple GEM amplifier. The peak position shown in Fig. 4 is related only to primary ionization 

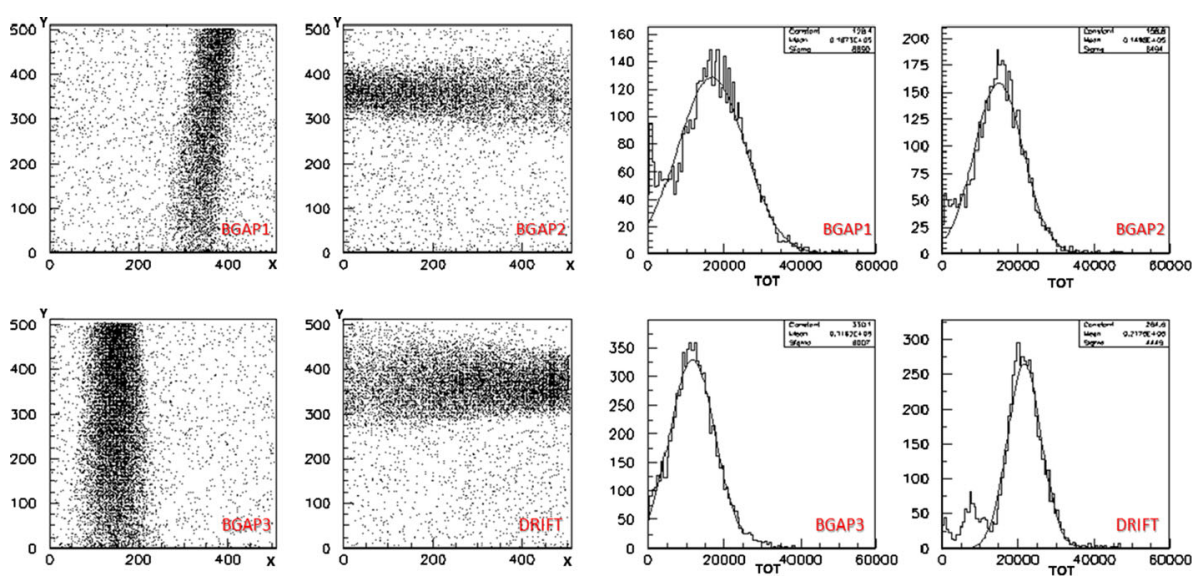

Fig. 3 On the left the 2D images of the X-ray flux entering in the different gap of the multi-boron converter. On the right the spectrum of the ${ }^{55} \mathrm{Fe}$ measured for each gap

Fig. 4 Reference charge deposited spectrum recorded when the detector is irradiated in the induction layer

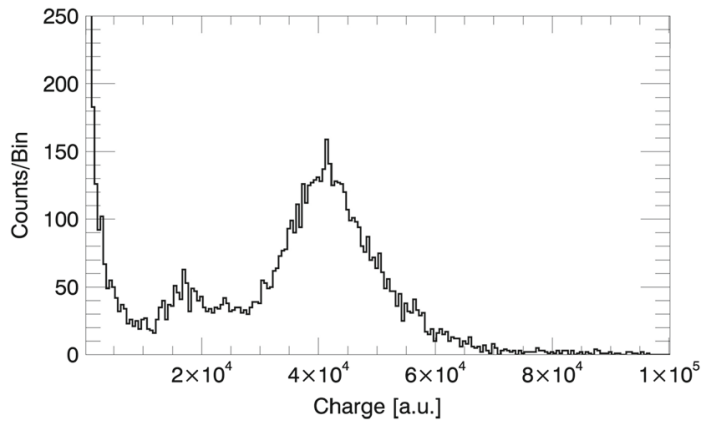

in the drift region and to triple GEM amplification. Therefore a change in the voltage applied to the converter will not affect the peak position.

The source was then moved around in order to irradiate regions between each BGEM one by one. This study allows to disentangle each single BGEM contribution. Each BGEM voltage is then tuned so that the position of main peak position obtained when the detector irradiated in the corresponding Bgap is comparable to the position obtained when X-rays convert only in the drift region. Figure 5 shows the recorded spectra obtained when the detector is irradiated in the different Bgaps with two different HV configuration ( $300 \mathrm{~V}$ and $330 \mathrm{~V}$ applied on each BGEM).

Each spectrum is fitted with a Gaussian to obtain the peak position $P_{\mathrm{Bgap}_{i}}$. The BGEMs potential differences are then tuned so that the charge extraction factor $C_{\mathrm{ex}_{i}}$ is equal to one for each irradiated Bgap volume:

$$
C_{\mathrm{ex}_{i}}=\frac{P_{\mathrm{Bgap}_{i}}}{P_{(\text {drift region })}} \approx 1
$$

Figure 6 shows the obtained coefficient values for the two HV configurations. The error bars represent the calculated FWHM of the fitted Gaussian.

The left panel shows that with a too low BGEM gain a significant fraction of the deposited charge is lost when the electrons have to transfer from one converter volume to the subsequent. 

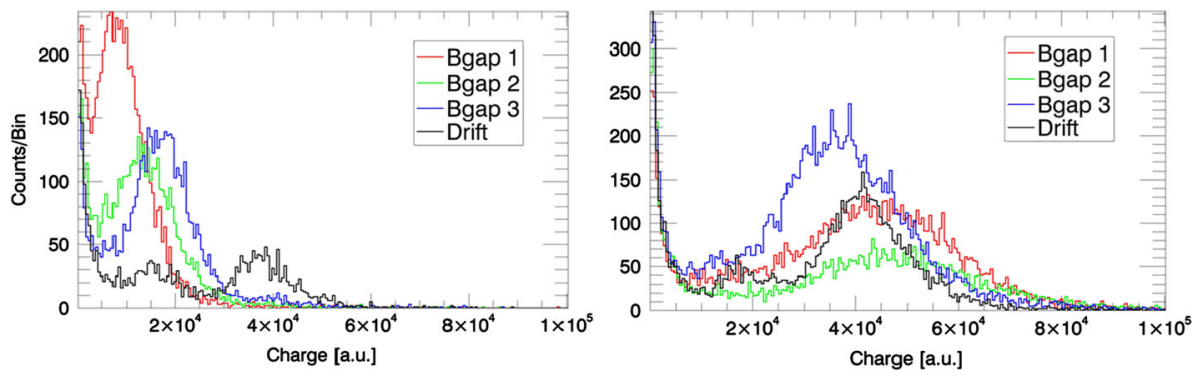

Fig. 5 Deposited charge spectrum recorded with the detector irradiated in the Bgap1 (red), Bgap2 (green), Bgap3 (blue) and drift region (black) with $\mathrm{BGEM}_{i}$ voltage $=300 \mathrm{~V}$ (left panel) and $330 \mathrm{~V}$ (right panel) applied on each BGEM

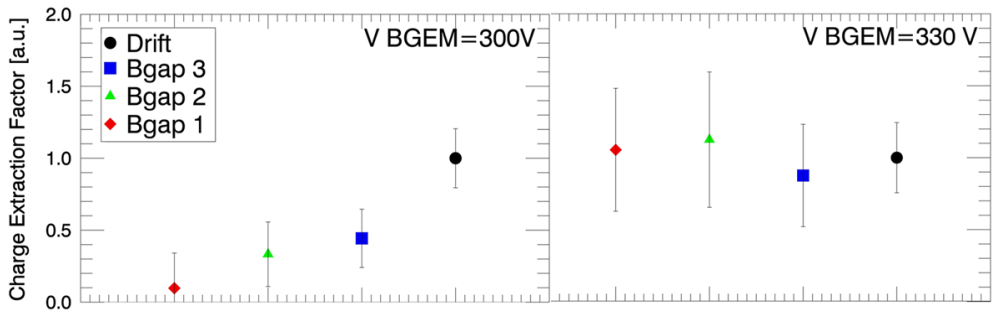

Fig. 6 Charge extraction factor for each $i_{\text {th }}$ irradiated volume of the converter obtained with $300 \mathrm{~V}$ (left panel) and $330 \mathrm{~V}$ (right panel) applied on each BGEM. The error bars represent the calculated FWHM

With the appropriate gain instead (right panel), the deposited charge is successfully transferred from one converter volume to another. Figure 6 shows also a degradation of the energy resolution (represented by the error bars) by increasing the distance of the irradiation point from the drift region. This is maybe due to the fact that a fraction of the created primary electrons is lost on the bottom electrode of each BGEM, but the average deposited charge (center position of the main peak) does not change because the lost electron fraction is replaced by the multiplication of the primary electrons that are able to enter in the BGEMs holes. The energy resolution degradation can be contained by acting on the transfer electric field present between two BGEM, and this optimization will be studied in the future. However, for counting application the energy resolution is not a crucial parameter, and the degradation shown by the 3 BGEM converter with the optimal found HV configuration suggests that the charge will be successfully transfer also in a converter with more than 3 BGEMs, giving the possibility to further increase the thermal neutron efficiency of the detector. The following $\gamma$-ray and neutron characterization was done with the converter $\mathrm{HV}$ configuration found in this study.

\subsection{Gamma ray characterization of the detector}

Gamma ray sensitivity is a crucial parameter for neutron detectors, given that they operate in an environment on which a gamma background due to neutron-induced reaction is always present. In a GEM detector gammas interact with the detector material mainly through Compton effect, producing a Compton electron that can give rise to a signal in the readout anode. Compton electrons travelling in the gas medium have a reduced stopping power producing elongated thin tracks typically with a low charge density. On the other hands, the ions pro- 


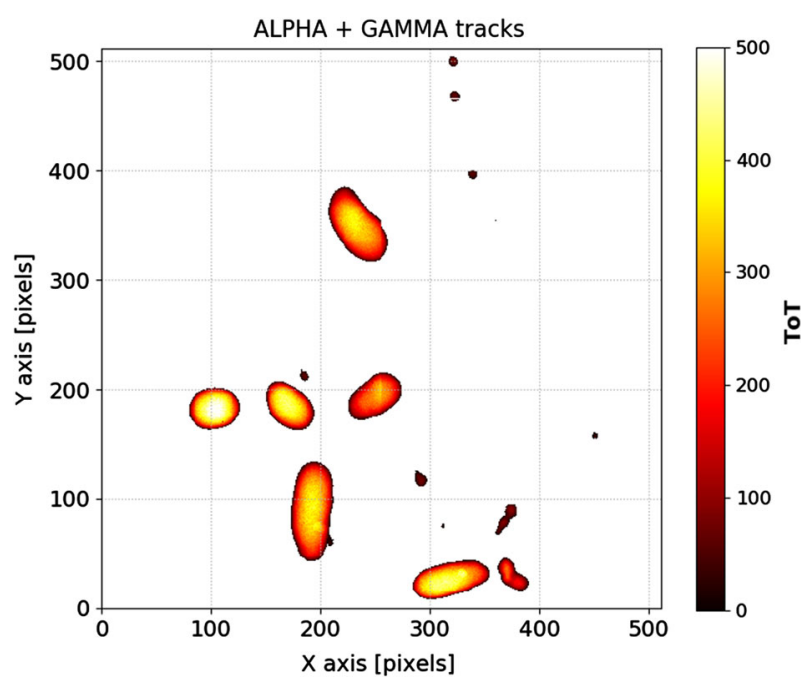

Fig. 7 The online display $\left(512 \times 512\right.$ pixels $\left.\simeq 3 \times 3 \mathrm{~cm}^{2}\right)$ showing six alphas produced by the thermal neutrons reaction and the small clusters produced by gammas (Compton electrons). The width of the clusters is clearly different between alphas and electrons

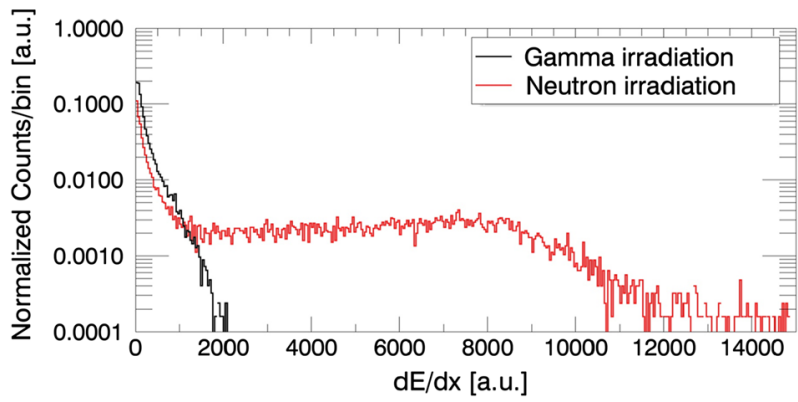

Fig. 8 Distribution of the $\mathrm{d} E / \mathrm{d} x$ factor recorded with the detector under gammas (black line) and neutron (red line) irradiation with $\mathrm{HVGem}=1240 \mathrm{~V}$

duced by the ${ }^{10} \mathrm{~B}(n, \alpha){ }^{7} \mathrm{Li}$ reaction have high stopping power in the gas medium, producing circular or elliptical tracks since they have a high charge density. Figure 7 shows an example of the recorded tracks for gammas and neutrons by the online display.

The different stopping power $(\mathrm{d} E / \mathrm{d} x)$ of the particles interacting with the detector gives a useful tool to perform an effective neutron/gamma discrimination. In fact, for each interacting particle the readout electronic gives both the total deposited charge and width of the produced track. The ratio between these two quantities gives an estimation of the stopping power. Figure 8 shows an example of the distributions of the $\mathrm{d} E / \mathrm{d} x$ factor obtained with the detector, respectively, under gammas (black line) and neutron (red line) irradiation.

Gamma sensitivity is strictly related to the detector gain, since the lower the gain, the less is the probability that a gamma will produce a trace capable of giving a signal over the threshold on the reading electronics. At the same time, at low gain even the charge produced by ions is lower, affecting the neutron detection efficiency of the detector. It is then crucial to determine a working point for the detector that maximizes the neutron detection efficiency minimizing 

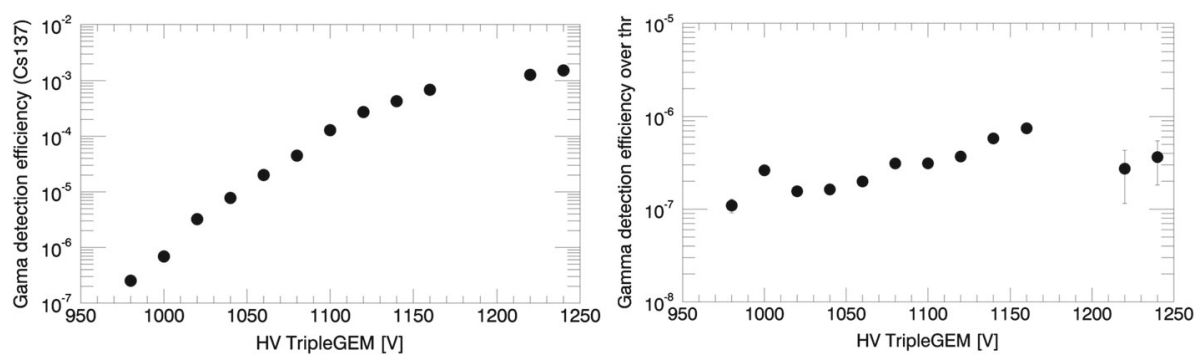

Fig. 9 Detection efficiency obtained for different detector gain under ${ }^{137} \mathrm{Cs} \gamma$-rays irradiation. On the left: no $\mathrm{d} E / \mathrm{d} x$ threshold applied. On the right: applying the set of $\mathrm{d} E / \mathrm{d} x$ thresholds

the gamma sensitivity. In order to do this, the detector was tested for different gains (applied to the triple GEM detector stage) using a ${ }^{137} \mathrm{Cs}$ source (662 $\mathrm{keV}$ photon energy). The gamma efficiency is determined by dividing the total number of recorded events (no threshold applied) with the estimated total flux of photons on the detector. The result of the scan is shown in Fig. 9.

As expected, the gamma sensitivity is an increasing function of the gain, but it is possible to determinate a set of $\mathrm{d} E / \mathrm{d} x$ thresholds (one threshold for each gain) so that the gamma sensitivity can be below a fixed value even for high gain. By applying the found set of $\mathrm{d} E / \mathrm{d} x$ threshold the gamma sensitivity obtained as a function of the gain is reported in Fig. 9.

The gamma detection efficiency obtained is less than $10^{-6}$ with the chosen set of threshold, allowing neutron measurements with a $n / \gamma$ discrimination factor better than $10^{5}$ assuming a neutron detection efficiency of about $10^{-1}$. The results shown in this paragraph highlight also that the detector performance is not influenced by the $478 \mathrm{keV} \gamma$-rays emitted during the ${ }^{10} \mathrm{~B}(n, \alpha){ }^{7} \mathrm{Li}$ when the ${ }^{7} \mathrm{Li}$ is left in the excited state since we expect a similar gamma sensitivity for $\gamma$-rays of this energy.

\subsection{Neutron characterization of the detector}

The detector was tested at the research nuclear reactor of Applied Nuclear Energy Laboratory (LENA) [30] of Pavia University, a 250-kW power Triga Mark II that offers different neutron irradiation positions; recently in one of the horizontal channels has been realized a collimated thermal neutron beam with a diameter of $49 \mathrm{~mm}$ and equipped with a system of shutters that allows the positioning of samples even when the reactor is in operation. The thermal flux at the exit of the channel is about $5 \times 10^{6} \mathrm{~cm}^{-2} \mathrm{~s}^{-1}$.

The neutron beam was collimated using a ${ }^{6} \mathrm{Li}$-enriched plastic sheet having a hole $(5 \mathrm{~mm}$ diameter) in the middle. The first test performed was a gain scan in order to determine the detector working point to be used for neutron detection (the minimum gain at which the neutron detection efficiency is maximized). In order to do this study, the same set of threshold found in the gamma scan of par. 3.2 was used. The result of the scan is shown in Fig. 10.

The recorded counting rate is an increasing function of the gain, showing a plateau for $\mathrm{HV} \approx 1120 \mathrm{~V}$, which represent the working point of the detector under neutron irradiation.

The neutron detection capability was measured as a function of the number of active converter layers in the detector. The BGEMs were initially set to $0 \mathrm{~V}$ (no charge transfer between different converter volumes), and the relative detection efficiency was measured by setting to $330 \mathrm{~V}$ each BGEM one by one. The relative detection efficiency is calculated as the ratio between the counting rate recorded with all the BGEM set to $0 \mathrm{~V}$ (with a single active 


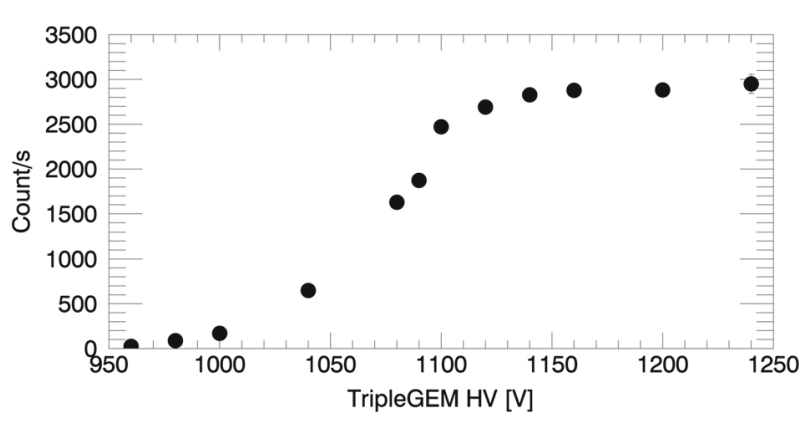

Fig. 10 Recorded over threshold counting rate as a function of the detector gain under thermal neutron irradiation
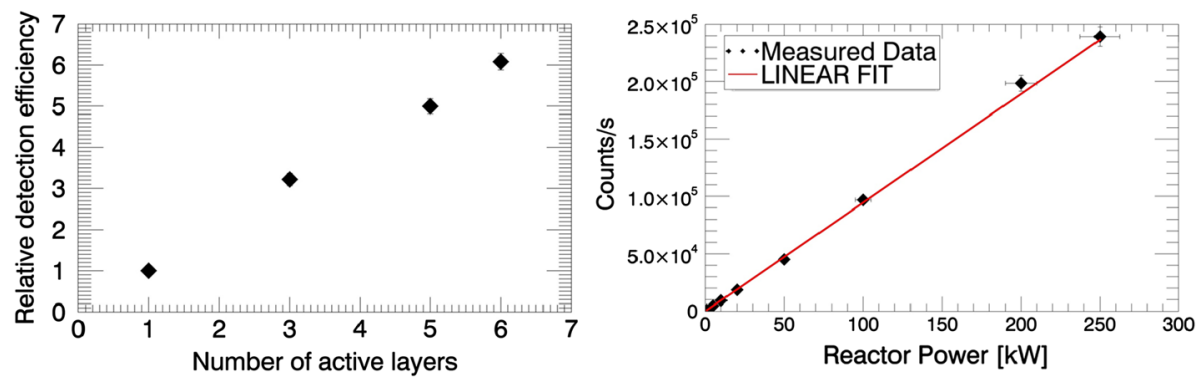

Fig. 11 On the left: relative detection efficiency measured as a function of the number of active converter layers. On the right: recorded counting rate on the detector as a function of the reactor power

converter layer) and the counting rate recorded with one, two or three BGEM set to $330 \mathrm{~V}$ (each active BGEM adds two active converter layers to the conversion stack). The result of this test is shown in Fig. 11, where the relative detection efficiency is reported as a function of the number of active converter layers.

As one can see, the measured relative neutron detection efficiency matches number of active converter layers, confirming the good charge extraction capability of the detector found in par. 3.1. The obtained results show that the detector is no lack of efficiency even when all the converter layers are active, giving the possibility to further increase the number of BGEMs and then to further increase the neutron detection efficiency. The last measurement was carried out in order to check the response of the detector under high neutron flux. The reactor power was varied from 1 to $250 \mathrm{~kW}$, and the detector was set to the best HV configuration with all the BGEMs active. Figure 11 shows the recorded counting rate on the detector as a function of the reactor power.

As one can see, the detector response is almost linear up to $250 \mathrm{kHz}$ corresponding to $250 \mathrm{~kW}$ of reactor power. This counting rate has been obtained with a neutron beam of $5 \mathrm{~mm}$ diameter that thus corresponds to a flux of $1.2 \mathrm{MHz} / \mathrm{cm}^{2}$. Further study, with a dedicated electronic readout for very high counting rate applications, will be carried out in the future in order to investigate the behavior of the detector in the very high counting rate region.

In order to estimate the expected neutron detection efficiency, simple calculations have been performed considering an average neutron energy $E_{n}=25 \mathrm{meV}$. The calculation is performed by using the Beer-Lambert law:

$$
I(E, x)=I_{0} e^{-n \sigma\left(E_{n}\right) x}
$$



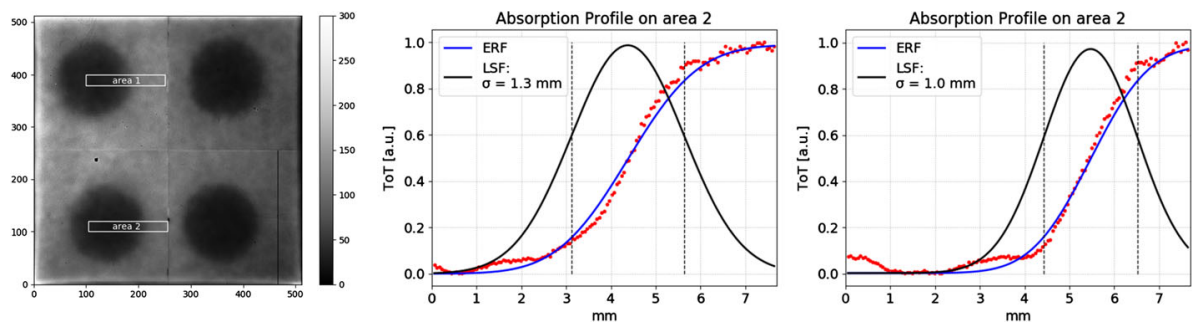

Fig. 12 On the left: the image obtained with 1-min exposition to the thermal neutron beam. On the right: the two profiles obtained from area 1 and area $2(140 \times 20$ pixels $)$ fitted with ERF and the corresponding LSF

where $I(E, x)$ is the transmitted intensity through a sample of density $n$ and thickness $x$, $I_{0}$ is intensity before the sample, and $\sigma\left(E_{n}\right)$ is the total neutron cross section. Therefore the absorbed neutron intensity is defined as $I_{0}-I=I_{0}\left(1-e^{-n \sigma\left(E_{n}\right) x}\right)$. By considering $\sigma\left(E_{n}=25 \mathrm{meV}\right)=3844 b, n=8.76 \times 10^{22}$ nuclei $/ \mathrm{cm}^{3}$ and $x=1 \mu \mathrm{m}$, a single layer absorption efficiency $p=3 \%$ is obtained. The escape probability $\xi$ for $x=1 \mu \mathrm{m}$ is about $65 \%$ [17], and the borated GEM foils have an optical transparency (ot) (ratio between empty and total area) of $78 \%$. Therefore a total efficiency expected for six ${ }^{10} \mathrm{~B}_{4} \mathrm{C}$ stacked layers can be defined as $\left(1-e^{-6 n \sigma\left(E_{n}\right) x}\right) \times \xi \times$ ot $\times \eta$. Assuming a unitary extraction efficiency $\eta$, the expected total efficiency is about $8.76 \%$. Further measurement will be performed in order to assess this value by using an absolutely calibrated ${ }^{3} \mathrm{He}$ tube.

\subsection{Spatial resolution}

A first estimate of the spatial resolution has been performed using four disks of cadmium having a thickness of $1 \mathrm{~mm}$ and a diameter of $8 \mathrm{~mm}$ placed just in front of the detector. Neutron absorption from cadmium produces circular shadow images (see Fig. 12) that are obtained integrating the total charge released by alphas and $\mathrm{Li}$ ions after neutron absorption and, to a lesser extent, to gammas produced by unstable nuclei. The image has been acquired with an high voltage of $1120 \mathrm{~V}$.

A measurement of the spatial resolution was obtained from the normalized edge response function (ERF) which has been calculated along the shadow edge profiles measured in area 1 and 2 as shown in Fig. 12. The length of this profile extends over 140 bins of $55 \mu \mathrm{m}$ each, the content of which is filled with the mean value on 20 pixels along the orthogonal direction. These normalized profiles have been fitted with the ERF, obtaining the corresponding derivative line spread function (LSF). According to Sparrow criterion, two shadow images can be considered distinct if their separation is equal to $2 \sigma$ of the LSF and this value can be considered a reasonable estimate of the spatial resolution ranging between 2.0 and $2.6 \mathrm{~mm}$.

\section{Conclusions and future work}

A first batch of GEM foils with boron coating $\left(1 \mu \mathrm{m}\right.$ thick $\left.{ }^{10} \mathrm{~B}_{4} \mathrm{C}\right)$ on both side was successfully realized and tested in a neutron detector equipped with a 3-layer converter readout by a GEMPix detector. All the six BGEMs produced passed the electrical tests, and three of them were used for the detector construction. The neutron characterization showed that in the optimized HV configuration, the MBGEM detector is able to increase the neutron detection efficiency obtained with a single layer of ${ }^{10} \mathrm{~B}_{4} \mathrm{C}$ by a factor directly proportional to the 
number of active converter foils, with a $n / \gamma$ discrimination factor better than $10^{5}$. With six layer of ${ }^{10} \mathrm{~B}_{4} \mathrm{C}$ the expected total efficiency is about $8.7 \%$. The detector response is almost linear up to $250 \mathrm{kHz}$ counting rate, corresponding to a flux of $1.2 \mathrm{MHz} / \mathrm{cm}^{2}$. A preliminary study showed a spatial resolution ranging between $2.0 \div 2.6 \mathrm{~mm}$.

The obtained results suggest the possibility to further increase the neutron detection efficiency by augmenting the number of borated GEM foils in the converter stack. The next step is the production of a bigger detector with an active area $10 \times 10 \mathrm{~cm}^{2}$ with an higher number of BGEM foils and read out by the GEMINI DAQ system [31], for high counting rate applications. This detector will also be characterized in terms of absolute neutron detection efficiency by comparing its performance to an absolutely calibrated ${ }^{3} \mathrm{He}$ tube. These measurements will be subject of a future publication.

Acknowledgements We wish to thank various people for their contribution to this detector; M. Silari, M. Cambell, for their valuable financial and technical support on the development of the GEMPix detector; G. Corradi and D. Tagnani for the design of the high- voltage and readout boards, and finally J. Alozy for the software and hardware support on Timepix chips. Support from the Swedish Research Council VRRFI (2017-00646-9) for the accelerator-based ion-technological center and from the Swedish Foundation for Strategic Research (Contract RIF14-0053) for the Tandem Accelerator Laboratory in Uppsala is gratefully acknowledged.

Funding Open Access funding provided by CERN.

Open Access This article is licensed under a Creative Commons Attribution 4.0 International License, which permits use, sharing, adaptation, distribution and reproduction in any medium or format, as long as you give appropriate credit to the original author(s) and the source, provide a link to the Creative Commons licence, and indicate if changes were made. The images or other third party material in this article are included in the article's Creative Commons licence, unless indicated otherwise in a credit line to the material. If material is not included in the article's Creative Commons licence and your intended use is not permitted by statutory regulation or exceeds the permitted use, you will need to obtain permission directly from the copyright holder. To view a copy of this licence, visit http://creativecommons.org/licenses/by/4.0/.

\section{References}

1. F. Sauli, Nucl. Instrum. Methods Phys. Res. Sect. A Accel. Spectrom. Detect. Assoc. Equip. 386, 531 (1997)

2. F. Murtas, Radiat. Meas. 138, 106421 (2020)

3. R.T. Kouzes, OSTI.GOV: Technical Report (2009)

4. G.F. Knoll, Radiation Detection and Measurement (Wiley, New York, 2010)

5. G. Bencivenni, G. Felici, F. Murtas, P. Valente, W. Bonivento, A. Cardini, A. Lai, D. Pinci, B. Saitta, C. Bosio, Nucl. Instrum. Methods Phys. Res. Sect. A Accel. Spectrom. Detect. Assoc. Equip. 488, 493 (2002)

6. G. Croci, G. Claps, M. Cavenago, M. Dalla Palma, G. Grosso, F. Murtas, R. Pasqualotto, E. Perelli Cippo, A. Pietropaolo, M. Rebai et al., Nucl. Instrum. Methods Phys. Res. Sect. A Accel. Spectrom. Detect. Assoc. Equip. 720, 144 (2013), selected papers from the 2nd International Conference Frontiers in Diagnostic Technologies (ICFDT2)

7. G. Croci, M. Rebai, G. Claps, M. Cavenago, M.D. Palma, G. Gervasini, G. Grosso, F. Murtas, R. Pasqualotto, E.P. Cippo et al., J. Instrum. 7, C03010 (2012)

8. F. Murtas, G. Croci, A. Pietropaolo, G. Claps, C.D. Frost, E.P. Cippo, D. Raspino, M. Rebai, N.J. Rhodes, E.M. Schooneveld et al., J. Instrum. 7, P07021 (2012)

9. A. Muraro, G. Croci, G. Albani, G. Claps, M. Cavenago, C. Cazzaniga, M. Dalla Palma, G. Grosso, F. Murtas, R. Pasqualotto et al., Nucl. Instrum. Methods Phys. Res. Sect. A Accel. Spectrom. Detect. Assoc. Equip. 813, 147 (2016)

10. M. Klein, C.J. Schmidt, Nucl. Instrum. Methods Phys. Res. Sect. A Accel. Spectrom. Detect. Assoc. Equip. 628, 9 (2011) 
11. G. Croci, G. Claps, R. Caniello, C. Cazzaniga, G. Grosso, F. Murtas, M. Tardocchi, E. Vassallo, G. Gorini, C. Horstmann et al., Nucl. Instrum. Methods Phys. Res. Sect. A Accel. Spectrom. Detect. Assoc. Equip. 732, 217 (2013), Vienna Conference on Instrumentation 2013

12. G. Croci, A. Muraro, E.P. Cippo, M. Tardocchi, G. Grosso, G. Albani, G. Angella, I. Defendi, R. HallWilton, C. Höglund et al., EPL (Europhys. Lett.) 123, 52001 (2018)

13. A. Muraro, G. Croci, E. Perelli Cippo, G. Grosso, C. Höglund, G. Albani, R. Hall-Wilton, K. Kanaki, F. Murtas, D. Raspino et al., Prog. Theor. Exp. Phys. 2018, 023 H01 (2018)

14. A. Pietropaolo, G. Claps, A. Fedrigo, F. Grazzi, C. Hoglund, F. Murtas, A. Scherillo, S. Schmidt, E.M. Schooneveld, EPL 121, 62001-1-62001-4 (2018)

15. A. Curioni, N. Dinar, F. La Torre, J. Leidner, F. Murtas, S. Puddu, M. Silari, Nucl. Instrum. Methods Phys. Res. Sect. A Accel. Spectrom. Detect. Assoc. Equip. 849, 60 (2017)

16. G. Claps, D. Pacella, F. Murtas, K. Jakubowska, G. Boutoux, F. Burgy, J.E. Ducret, D. Batani, Rev. Sci. Instrum. 87, 103505 (2016). https://doi.org/10.1063/1.4964731

17. C. Hoglund, J. Birch, K. Andersen, T. Bigault, J.C. Buffet, J. Correa, P. Van Esch, B. Guerard, R. HallWilton, J. Jensen et al., J. Appl. Phys. 111, 104908-1-104908-8 (2012)

18. F. Piscitelli, P.V. Esch, J. Instrum. 8, P04020 (2013)

19. R. de Oliveira (2010), https://kt.cern/technologies/gas-electron-multiplier. Accessed 2021 CERN

20. C. Höglund, K. Zeitelhack, P. Kudejova, J. Jensen, G. Greczynski, J. Lu, L. Hultman, J. Birch, R. HallWilton, Radiat. Phys. Chem. 113, 14 (2015)

21. S. Schmidt, C. Hoglund, J. Jensen, L. Hultman, J. Birch, R. Hall-Wilton, J. Mater. Sci. 51, 10418 (2016)

22. Y. Zhang, H.J. Whitlow, T. Winzell, I.F. Bubb, T. Sajavaara, K. Arstila, J. Keinonen, Nucl. Instrum. Methods Phys. Res. Sect. B Beam Interact. Mater. Atoms 149, 477 (1999)

23. M. Moro, R. Holeňák, L. Zendejas Medina, U. Jansson, D. Primetzhofer, Thin Solid Films 686, 137416 (2019)

24. H.Y. Qu, D. Primetzhofer, M.A. Arvizu, Z. Qiu, U. Cindemir, C.G. Granqvist, G.A. Niklasson, A.C.S. Appl, Mater. Interfaces 9, 42420 (2017)

25. CAEN (2019). https://www.caen.it/products/A1561h/. Accessed 2021 CAEN

26. G. Corradi, F. Murtas, D. Tagnani, Nucl. Instrum. Methods Phys. Res. Sect. A Accel. Spectrom. Detect. Assoc. Equip. 572, 96 (2007), frontier Detectors for Frontier Physics

27. X. Llopart, R. Ballabriga, M. Campbell, L. Tlustos, W. Wong, Nucl. Instrum. Methods Phys. Res. Sect. A Accel. Spectrom. Detect. Assoc. Equip. 581, 485 (2007)

28. V. Kraus, M. Holik, J. Jakubek, M. Kroupa, P. Soukup, Z. Vykydal, J. Instrum. 6, C01079 (2011)

29. D. Turecek, T. Holy, J. Jakubek, S. Pospisil, Z. Vykydal, J. Instrum. 6, C01046 (2011)

30. LENA (2010). https://lena.unipv.it/en/homepage/. Accessed 2021 LENA

31. A. Pezzotta, G. Corradi, G. Croci, M. De Matteis, F. Murtas, G. Gorini, A. Baschirotto, GEMINI: A TripleGEM Detector Read-Out Mixed-Signal ASIC in $180 \mathrm{~nm}$ CMOS, in 2015 IEEE International Symposium on Circuits and Systems (ISCAS), IEEE (IEEE, 345 E 47th St, New York, NY 10017 USA, 2015), IEEE International Symposium on Circuits and Systems, pp. 1718-1721, ISBN 978-1-4799-8391-9, ISSN 0271-4302, IEEE International Symposium on Circuits and Systems (ISCAS), Lisbon, PORTUGAL, MAY 24-27, 2015 